\title{
A new method to detect circles in images based on genetic algorithms
}

\author{
Navid Khalili Dizaji ${ }^{1,}$, , Nazila Masoudi ${ }^{1}$, Aidin Sakhvati ${ }^{2}$ \\ ${ }^{1}$ Department of Mechatronics Engineering, Tabriz Branch, Islamic Azad University, Tabriz, Iran \\ ${ }^{2}$ Department of Electrical Engineering, Tabriz Branch, Islamic Azad University, Tabriz, Iran
}

Email address:

navidkhalili@yahoo.com (Navid K. D.), nazila_masudi@yahoo.com (Nazila M.), aidin_sakhavati@yahoo.com (Aidin S.)

To cite this article:

Navid Khalili Dizaji, Nazila Masoudi, Aidin Sakhvat. A new Method to Detect Circles in Images Based on Genetic Algorithms. International Journal of Intelligent Information Systems. Special Issue: Research and Practices in Information Systems and Technologies in Developing Countries. Vol. 3, No. 6-1, 2014, pp. 49-55. doi: 10.11648/j.ijiis.s.2014030601.19

\begin{abstract}
Object detection is one of the key issues in digital image processing. Over the years, many algorithms have been created for detecting meaningful objects on the image which are based on specific characteristics of object or complex mathematical methods. Circle detection is one of these types of methods. One of the best methods for circle detection on digital images and discussion of machine vision is the Hough transform. The Hough transform can be described as the transformation of a point in the $\mathrm{x}$-y-plane to the parameter space. Parameter space can be defined by the shape of the object. Using the special character of each image in space, we are able to retrieve and extract the image circle. Importantly, this method is time consuming and a large amount of memory is required for the image. The undesirable features have reduced the popularity of this method. The idea of using genetic algorithm for detecting a circle in the picture is very attractive and functional. This method can be used in Robot Soccer, targeting systems and iris recognition. In this method, accuracy and speed are among important parameters. For example, in the case of robot, it should detect ball in monochromatic and sometimes crowded areas (due to accumulation of other bots around the ball). Using a genetic algorithm for circle detection on images, Hough transform weaknesses have been removed. It also increases the computation speed and accurate detection of circle. In this paper, the Hough transform method will be presented and then we will describe the process of implementing genetic algorithms to find a circle in the picture.
\end{abstract}

Keywords: Hough Transform, Image Processing, Digital Image

\section{Introduction}

A common problem in determining the location is the number or the orientation of specific objects in image. For example, it can be the determination of direct roads in aerial imagery. This problem can be solved using the Hough transform for these lines. Most interesting, objects have other forms such as circle, oval or any other shape. Despite the enhancement of Hough transform complexity along with increasing the number of parameters required to describe the desired shape, Hough ordinary transform can be used on any shapes [2].

Circular Hough transform algorithm is summarized as follows [2]:

1 Find the edges

2 For each edge point "Hough transform begins»"

3 A circle of radius $r$ centered at the edge point is drawn and all the coordinates the circumference of the circle passes will be increased.

4 Maximum points are found in the storage. "The Hough transform will be finished"

5 Found parameters $(\mathrm{r}, \mathrm{a}, \mathrm{b})$ are determined on the original image in accordance to the maximum points.

\section{Hough Transform for Circle Detection}

\subsection{Parametric Display}

The Hough transform is described as the transformation of a point in the $x-y$-plane to the parameter space. Parameter space can be defined by the shape of the object. A straight line which passes the points of $(x 1, x 1)$ and $(x 2, y 2)$ in the $x-y-p l a n e$ is defined as follows: 


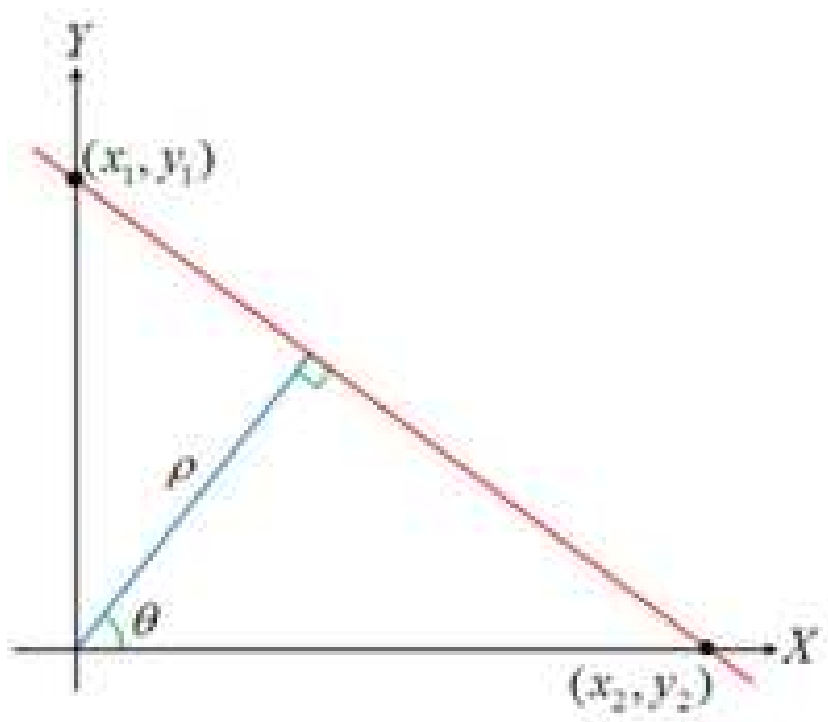

Figure 1. Showing a line in Cartesian coordinates

The relation is an equation for the straight line in a Cartesian coordinate system where ' $a$ ' and ' $b$ ' are the line parameters. Since the line perpendicular to the vector $x$ has an infinite value leading to infinite volume of ' $a$ ' and ' $b$ ', the Hough transform of line does not use this relation. Instead of displaying the line as above, the line the parameter space can be displayed as below:

$$
\begin{gathered}
\left\{\begin{array}{c}
a=-\frac{\cos \theta}{\sin \theta} \\
\mathrm{b}=\frac{\rho}{\sin \theta}
\end{array} \Rightarrow \mathrm{y}=\left(-\frac{\cos \theta}{\sin \theta}\right) \mathrm{x}+\left(\frac{\rho}{\sin \theta}\right)\right. \\
\rightarrow \rho=\mathrm{x} \cdot \cos \theta+\mathrm{y} \cdot \sin \theta
\end{gathered}
$$

Equation 1. Parameter space

In this case, the parameter space includes and which is called limited volume

$$
r^{2}=(x-a)^{2}+(y-b)^{2}
$$

Equation 2. limited volume

Circle in Cartesian coordinates can be expressed as follows:

$$
\begin{aligned}
& x=a+r \cdot \cos \theta \\
& \mathrm{y}=\mathrm{b}+\mathrm{r} \cdot \sin \theta
\end{aligned}
$$

Equation 3. Circle in Cartesian coordinates

Compared to lines, circles in parameter space can be expressed more easily because circle parameters can be directly sent to the parameter space. In the above equation, ' $a$ ' and ' $b$ ' are coordinates of the circle center along the center line of ' $x$ ' and ' $y$ '. ' $r$ ' is the radius of the circle. Parametric display of above equation is as follows:

It is clear that the parameter space of the circle is and the parameter space of the line is . With increasing the number of parameters required to describe the desired shape and the $\mathrm{R}$ size enhancement of the parameter space, the Hough transform complexity increases. To simplify the parameter space of the circle, the radius is recorded as a fixed number in this space [2].

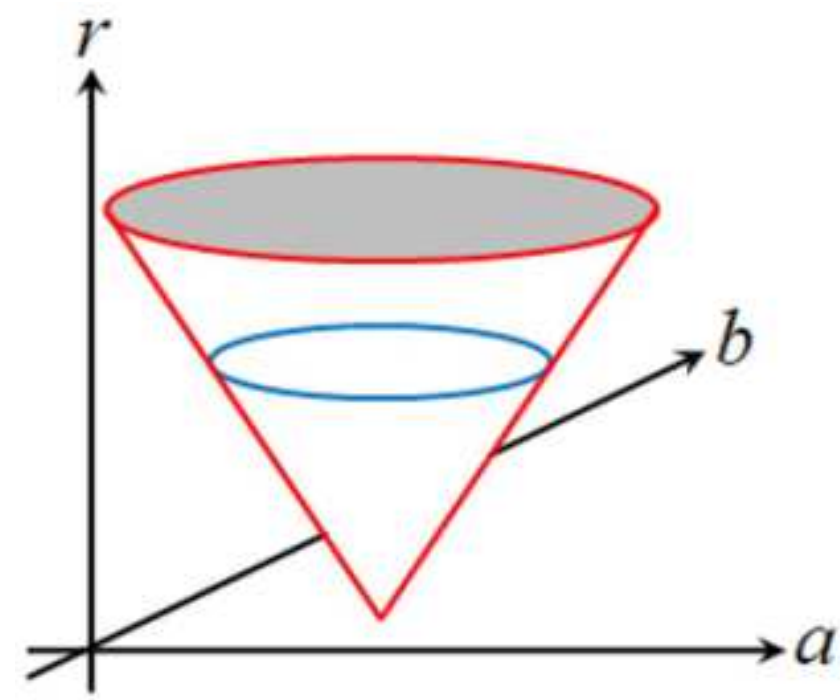

Figure 2. The parameter space of the circle after the Hough transformation

\subsection{Storage}

The process of finding the circle in an image using Hough transform is as follows:

First of all, all edges in the image are identified. It has no relevance to the Hough transform and all preferred technique of edge detection such as Sobel and Kenny can be used [3].

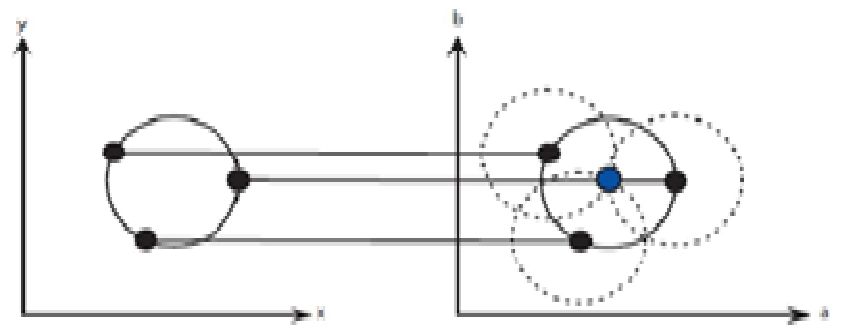

Figure 3. Conversion of a circle from the $x-y$ area (left) to the parameter space (right) for constant radius

Furthermore, at each edge point, a circle centered at that point is drawn with desired radius. This circle is drawn in the parameter space in such a way that the $\mathrm{x}$-axis, $\mathrm{y}$-axis and $\mathrm{z}$-axis model the components of ' $\mathrm{a}$ ', ' $\mathrm{b}$ ' and circle radius respectively. In coordinates which are drawn for the circumference of the circle, matrix storage value, which has equal sizes with parameters space, is increased. In this way, all edge points of the original image are investigated through drawing the circles with desired radius and increasing amounts in the storage. After that, the storage contains numbers presenting the number of passing circles from a unique coordinate. Therefore, the larger numbers which are selected intelligently based on the radius, correspond to the centers of the circles in the original image. 
US $5 \mathrm{ct}$ and $1 \mathrm{ctcoins}$

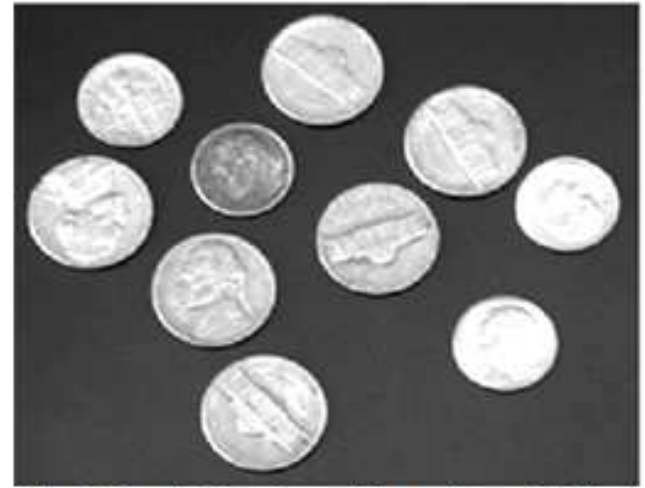

Circle Hough Transform with a radius of 25 pixels

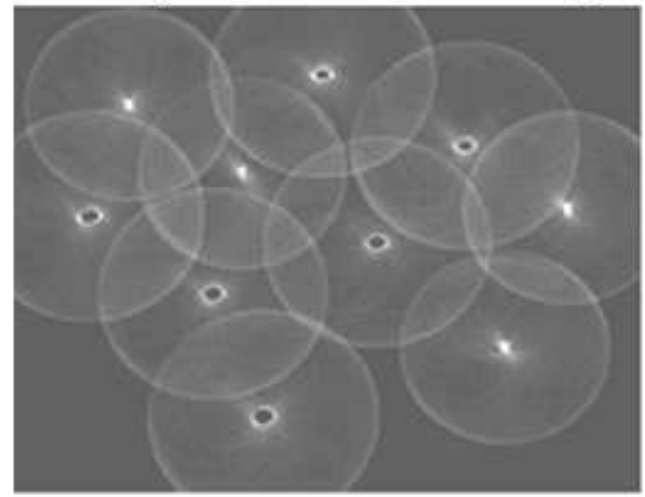

Edge streng ths from Sobel filtering

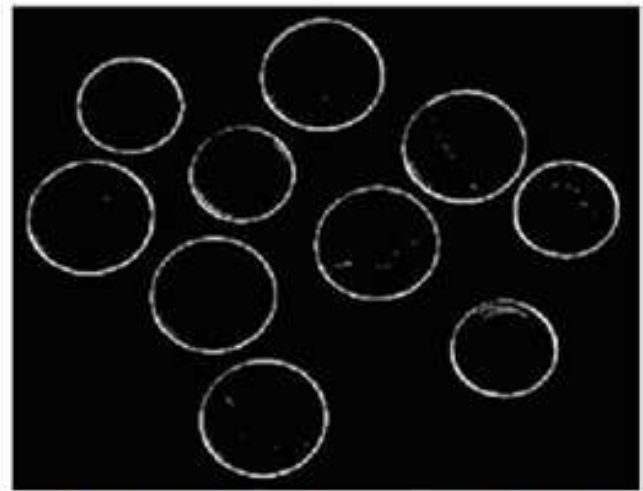

Circle Hough Transform with a radius of 30 pixels

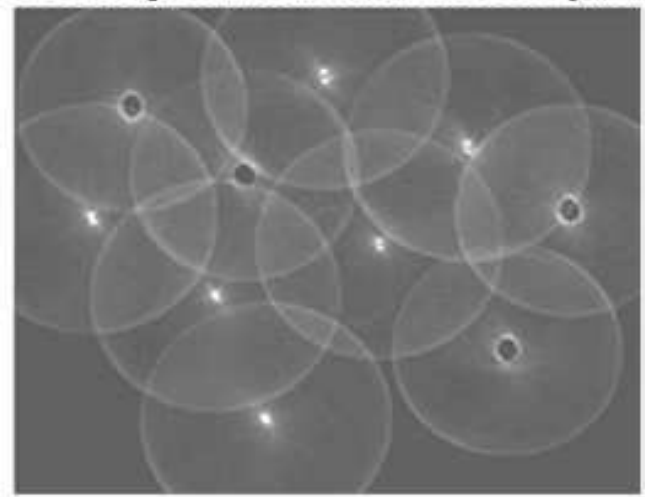

Figure 4. Example of the storage operation using actual data which shows the Hough transform for two radius values

\subsection{Hough Transformation Algorithm}

The process flow of Hough transformation algorithm is enumerated as below:

I. Find the edges

II. For each edge point "Hough transform begins»)

III. A circle of radius $r$ centered at the edge point is drawn and all the coordinates the circumference of the circle passes will be increased.

IV. Maximum points are found in the storage. "The Hough transform will be finished"

V. Found parameters ( $r, a, b)$ are determined on the original image in accordance to the maximum points.

\section{Implementation}

According to the algorithm presented in the previous section, the algorithm implementation can be conducted. However, before this, few points should be considered.

\subsection{How can the Data be Stored}

F If the radius is not fixed, the three-dimensional storage array can grow very fast in terms of stored data. The size of this array depends on the number of radius values and more importantly the size of the image. The computational cost to compute all the circles associated with the edge points will increase with the edge point's enhancement. The number of edge points is usually a function of image size. The calculation of total time of Hough transform for circles of large images with a lot of edge points can reach the unbridled amount of time rapidly.

\subsection{How to Draw a Circle in the Discrete Space}

A circle in the discrete space can be drawn using the Equation 3. However, the problem is. How to choose the discrete values with resolution of ? One solution is to use high resolution of and make the values rounded. But it may lead to draw the edge pixels more than once or, in the case of large radius values, it may lead to lack of pixels. The other solution is to round sine and cosine after multiplying the values by radius.

To ensure that all pixels are drawn, resolution of must be high. This will increase the computational cost. One method that can reduce computing cost is the quick calculation of values for sine and cosine functions using a lookup table. Although the discussed methods are feasible, but there are still better solutions [4].

Instead of using Equation 3, Bresenham's algorithm [4] can be used to draw a circle. The algorithm is designed to draw a line or a circle for a digital monitor. This algorithm has no problem in drawing a pixel more than once and it is appropriate for circular Hough transformation. An interesting feature of this algorithm is the determination of the pixels' actual number used to draw the circle. This information is 
appropriate when the center of the circle is to be found from the storage data.

\subsection{How can the Circle be Found from the Hough Transform Data}

Although this discussion is not related to the Hough transform, but it is useful for circles information extraction from the storage data. If there is no information about the number of circles and their radiuses, this process will be very cumbersome. One approach is to find the peaks of $a$ and $b$ planes each corresponding to a specific radius as shown in Figure 5.

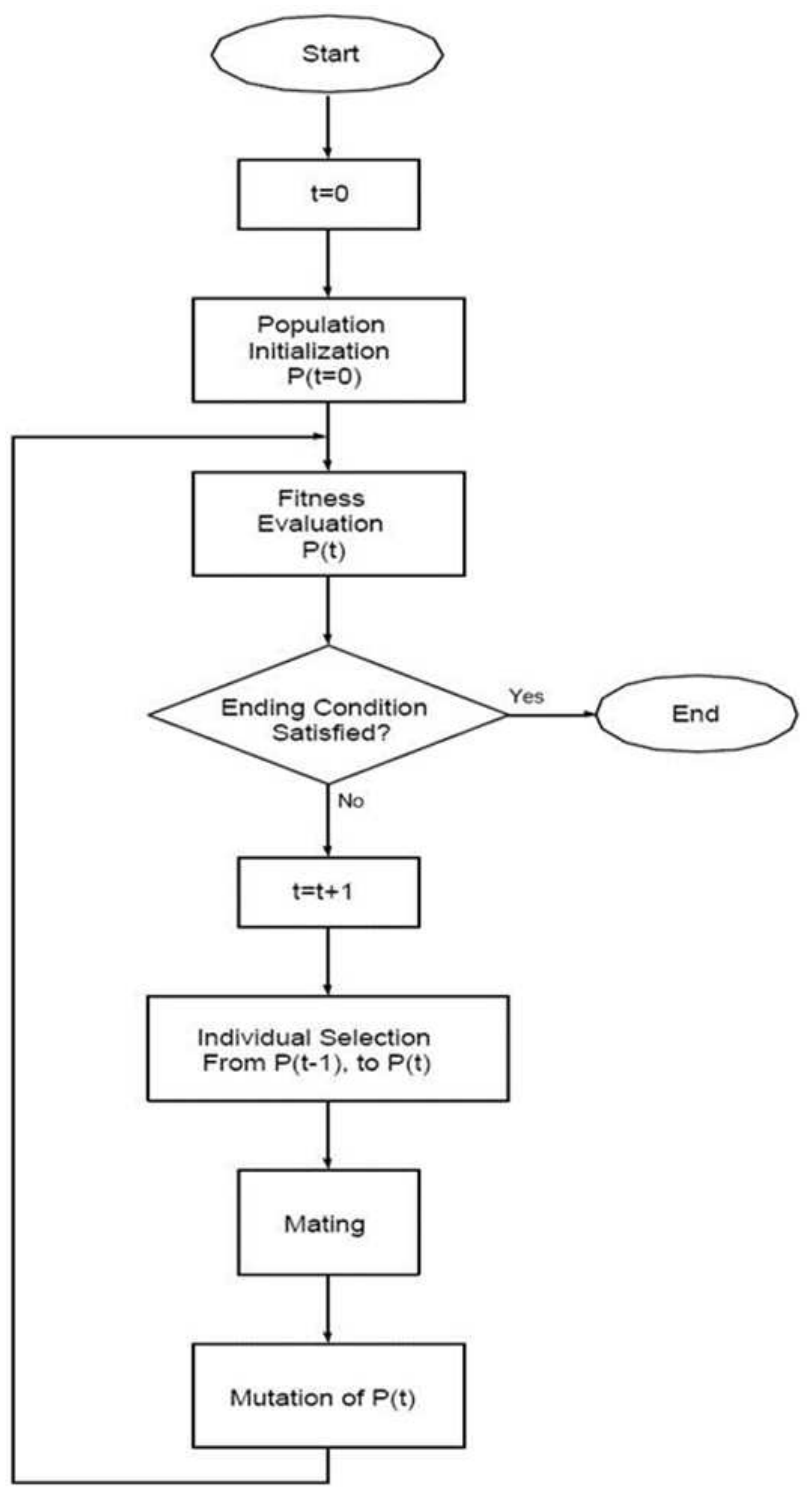

Figure 5. Block diagram of a simple Genetic Algorithm (SGA)

If the height of the peaks are equal compared to the number of edge pixels of the circle with a certain radius, peaks coordinates may correspond to the center of this circles. In the case of incomplete circle or oval shape, the center of the circle can be displayed with a height less than the number of the edge pixels. Eventually, if there is any difficulty in peaks determining, data storage can be paved.

\section{Genetic Algorithm}

Genetic Algorithm (GA) is a searching technique in computer science and artificial intelligence to find the optimal solution for the searching issues [5]. Genetic Algorithm is one of the Evolutionary Algorithms which is inspired by science of biology such as inheritance, mutation, sudden selection, natural selection and composition. It is briefly stated that Genetic Algorithm (GA) is a programming technique which uses genetic evolution as a model of problem solving. Most of the time, when the term of "struggle for survival" is used, its negative value comes to our mind. However, to rest assured, you can think that strongest was not always the winner. Despite their massive size and power, dinosaurs have ceded the survival game and having generation during a quite natural process, while much weaker creatures have continued their lives. It seems that the nature chooses the bests not only based on the size. In fact, it is more correct to say the nature chooses the fittest not the best. In the law of natural selection, having descendant is limited to some population's species which have the best features. Those who do not possess these characteristics will gradually disappear over time. For example, suppose a certain kind of people which are much more intelligent than the rest of the colony. In normal conditions, these people will improve more and have a relatively higher welfare. This welfare will lead to longer life and better reproduction. If intelligence is inheritable, the number of intelligent offspring will be more in the next generation of that community. If this trend continues, you will see that our sample population becomes smarter over the generations. In this way, a natural simple mechanism has managed to remove low-intelligent people of community. In addition, the average intelligence of community is constantly increasing [5].

Thus, we can see that the nature is able to improve each generation in terms of different features, using a simple mechanism; gradual elimination of undesirable species and reproduction of optimal species. The solutions are typically represented as binary 0 and 1 , but there are other ways to display it. Evolution is started from a random set of entities and repeated in subsequent generations. In each generation, the fittest is selected not the best. One solution to this problem is shown by a list of parameters called chromosome or genome. Generally, chromosomes are displayed as a simple string of data. Other types of data structures can be used. At first, several features are produced randomly to create the first generation. During each generation, each feature is evaluated and the fitness value is calculated by fitness function. Typically the genetic algorithms have a number of possible connections which is between 0.6 and 1 . It indicates the possibility of children creation. Organisms are combined together with this possibility. Two chromosomes connection creates the child which is added to the next generation. These are done until the appropriate candidates for response in the generation are emerged. The next step is 
to change the new children. Genetic algorithms have a fixed and small transition probability which is about 0.2 or less. Based on this possibility, child chromosomes are randomly changed or mutated.

The next step is to create a second generation of community. It is conducted by genetic operators (Chromosomes connected to each other and changed) based on the selection processes and production of selected features For each individual, a pair of parents is selected. The selections are in such a way that the best elements are selected so that the weakest elements have the chance to be selected to prevent achieving the local response. This process creates a new generation of chromosomes which is different from previous generation. The whole process is repeated for the next generation. Pairs are selected for combination. The third generation population comes to existence and so on.

This process is repeated until we reach the last stage.

Terminating conditions of genetic algorithms include [5]

1 To reach a fixed number of generations.

2 Allocated funds to be finished (computation time / money).

3 One person (the produced child) to be found who meet minimum (lowest) criteria.

4 To reach the highest degree of children process and not to yield better results.

5 Manual inspection.

6 High combinations.

Figure 5 shows a flowchart of a simple Genetic Algorithm (SGA) [5].

Stored as a binary two level matrix. To extract the edges with the width of a pixel, Gaussian edge detection.

Figure 6 shows the Gaussian operator output for a gray image $[6,7]$.
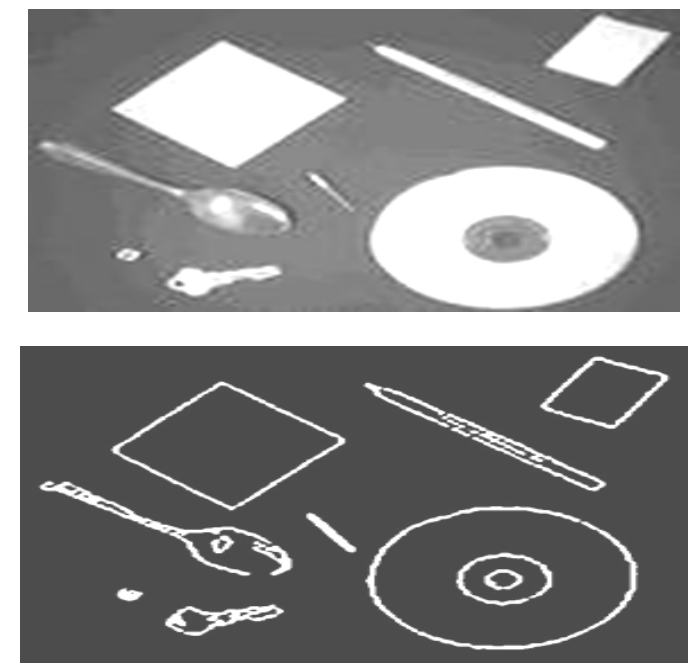

Figure 6. Input image (upper) and a binary image of the Gaussian edge detection operator

\section{Circle Detection Using the Genetic Algorithm}

According to the quadratic equation, each circle can be passed from three points in the image edge space. In simple terms, given the coordinates of three points, we can make a circle passing through it and extract the circle equation (If these three points have the potential to form a circle and not to be in a row). Preprocessed images have been used in our study. These images have been extracted by MATLAB and All edge points of the binary image should be stored in an array called $\mathrm{v}$, with the following structure.

$$
\begin{gathered}
\mathrm{V}=\left\{\mathrm{V}_{-} 1, \mathrm{~V}_{-} 2, \ldots, \mathrm{V}_{-} \mathrm{m}\right\} \\
\text { Equation 4. Edage array }
\end{gathered}
$$

Where ' $\mathrm{m}$ ' is the total number of edge points in the image. It should be noted that the number of edge pixels in the image varies in accordance to the image size and texture. When listing the Edge values in the array $\mathrm{V}$, the coordinates $\left(\mathrm{x}_{\mathrm{i}}, \mathrm{y}_{\mathrm{i}}\right)$ of each edge pixel Vi should be stored in the edge vector. By changing any addresses form 1 to ' $\mathrm{m}$ ' to a binary form and using three indicators of $i_{1}, i_{2}$ and $i_{3}$ for the edge points, the circle passing through the three points $V_{i 1}$ and $V_{i 2}$ and $V_{i 3}$ has the potential to detect circle in the image. A number of triple candidates which have the potential to solve circle is used for the initial population production in algorithm.

\subsection{Type of Representation}

Each individual circle can be revealed by three edge pixels on a chromosome (Figure 7). In this method, the address of edge points of the image in the genes is stored in accordance to it place in the edge array $\mathrm{V}$ (Figure 8). In this case, each chromosome has address of three edge points in the edge array $\mathrm{V}$ which are stored in three sequential genes.

$$
\left(\mathrm{x}-\mathrm{x}_{0}\right)^{2}+\left(\mathrm{y}-\mathrm{y}_{0}\right)^{2}=\mathrm{r}^{2}
$$

Equation 5. Genotype circle equation

\begin{tabular}{|l|l|l|}
\hline$v_{i}$ & $v_{j}$ & $v_{k}$ \\
\hline
\end{tabular}

Figure 7. A chromosome contains three gene to describe the circle edge pixel

$$
\begin{gathered}
x_{0}=\frac{\left|\begin{array}{ll}
x_{j}^{2}+y_{j}^{2}-\left(x_{i}^{2}+y_{i}^{2}\right) & 2\left(y_{j}-y_{i}\right) \\
x_{k}^{2}+y_{k}^{2}-\left(x_{i}^{2}+y_{i}^{2}\right) & 2\left(y_{k}-y_{i}\right)
\end{array}\right|}{4\left(\left(x_{j}-x_{i}\right)\left(y_{k}-y_{i}\right)-\left(x_{k}-x_{i}\right)\left(y_{j}-y_{i}\right)\right)} \\
\text { Equation 6. Transformations } X \\
y_{0}=\frac{\left[\begin{array}{ll}
2\left(x_{j}-x_{i}\right) & x_{j}^{2}+y_{j}^{2}-\left(x_{i}^{2}+y_{i}^{2}\right) \\
2\left(x_{k}-x_{i}\right) & x_{k}^{2}+y_{k}^{2}-\left(x_{i}^{2}+y_{i}^{2}\right)
\end{array}\right]}{4\left(\left(x_{j}-x_{i}\right)\left(y_{k}-y_{i}\right)-\left(x_{k}-x_{i}\right)\left(y_{j}-y_{i}\right)\right)}
\end{gathered}
$$

Equation 7. Transformations $Y$

According to the above equations, it can be called $\mathrm{T}$ transfer function with input edge indices of $\mathrm{i}, \mathrm{j}$ and $\mathrm{k}$. 


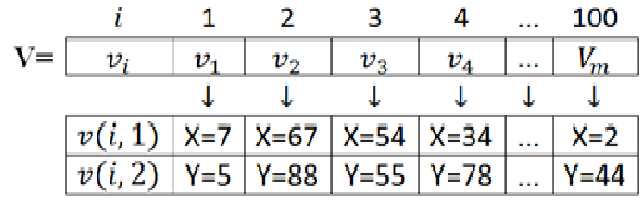

Figure 8. The number of each gene points out a cell address of the array $V$. The coordinates of the edge pixels are recorded in this cell.

Each circle is coded to three edge points of the image. The phenotype surface of each circle is indicated by three parameters of $\mathrm{X} 0$ and $\mathrm{Y} 0$ and $\mathrm{r}$. is coordinate of the center of the circle and $r$ is circle radius. For conversion of Genotype to Phenotype, the following equations are used. In fact, center and radius of the circle passing through the three edge points are estimated using the following equation [8].

$$
\left[\mathrm{x}_{0}, \mathrm{y}_{0}, \mathrm{r}\right]=T(i, j, k)
$$

Equation 8. Transfer function

With $\mathrm{T}$ transfer function, the circle of each chromosome in a discrete space can be easily estimated.

\subsection{Fitness Function}

To calculate the fitness of each chromosome, coordinates of the circle passing through the edge pixels of the chromosome should be computed using the $\mathrm{T}$ transfer function (equation 6 , 7 and 8). Actually we should obtain three parameters of, and $r$. Then, coordinates of the edge points in $\mathrm{V}$ should be computed. The number of these points is designated as test set and shown as. Function of is a number of Matrix V points which apply in the coordinates of the circle passing through three points. Our goal is to make the $\mathrm{C}$ value close to the maximum value. The larger the value, the better the answer for circle candidate is. The optimization process can be stopped due to the number of generations or the threshold value of the fitness. With regard to the issue, each of these methods can be employed. In this paper, the number of generations is the stop condition of the algorithm $[9,10]$.

\subsection{Selection Operator}

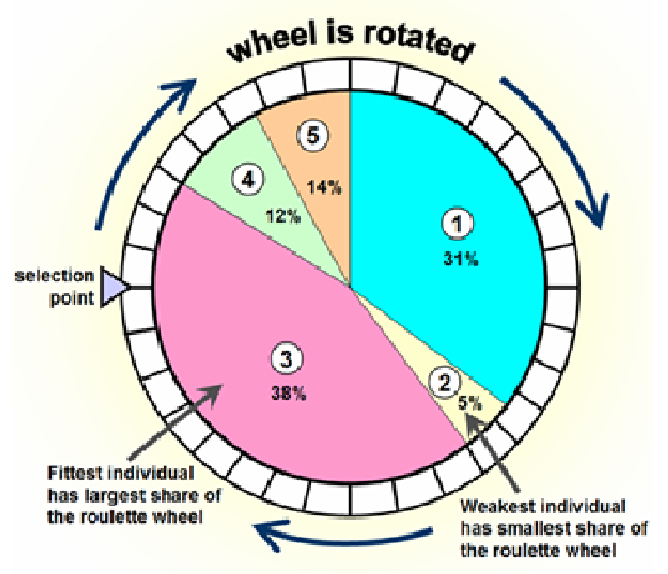

Figure 9. The Roulette Wheel [8]
At this stage, an appropriate number of paired chromosomes are selected based on their fitness level to be used in the next stage. Chromosomes with high fitness values can be selected several times during the production phase. Chromosomes with low fitness values may not be selected. For this step, the selection method of random pairs is used as a roulette wheel model of weighted ranking. In this model, the surface of wheel is divided into segments whose number is equal to the population members. The surface of each section is appropriate to the fitness value of each chromosome. Then the wheels circulate to be stopped in a point accidentally. This point specifies the selected chromosome. Figure 9 shows the roulette wheel.

This type of selection increases the number of optimal chromosomes in the population over time so that the average fitness value of the population increased compared to the past population.

\subsection{Blending Operator}

At this stage, cross operator acts on the parent chromosomes with Pc probability and produces new chromosomes with their combinations. In this paper, the single point blending is used.

\subsection{Mutation Operator}

In this paper, the mutation rate operator of $20 \%$ is used. In this step, the mutation is done on displacement chromosome with Pm probability. Each chromosome is randomly selected with a same probability and a random chromosome of $\mathrm{V}$ collection is substituted.

The following table shows the parameter values of the genetic algorithm for circle detection problem [10].

Table 1. Parameters of the genetic algorithm for circle detection problem in an image

\begin{tabular}{ll}
\hline Parameter & Value \\
\hline Population size & 100 \\
Crossover probability & 0.5 \\
Mutation probability & 0.2 \\
Number of elite individuals & 2 \\
Selection method & Roulette wheel \\
Crossover method & Crossover \\
Population size & 100 \\
\hline
\end{tabular}

\section{Discussion and Conclusion}

In this paper, a method for detecting objects in images is proposed. To this end, the characteristics of the Hough transform method and genetic algorithm classifier are used. With improving the estimation method of initial points, a higher percentage of detection can be achieved. Another important issue is the dependence of the classification capabilities of the network to the collection combination of training images. For example, with a big number of images, the amount of output neurons will be reduced and it causes the output to be less than the threshold value in the images. In this regard, we can study the composition of the sample. The feature selection can also 
help to improve process because, in this method, all features of feature extraction phase have been used.

In some cases, where the preprocessing is done in windows with linear functions, weights convolution of the input layer of genetic algorithm with image can be used to speed up.

\section{References}

[1] Tahir Rabbani and Frank van den Heuvel,"Efficient Hough transform for automatic detection of cylinders in point clouds." in Proceedings of the 11th Annual Conference of the Advanced School for Computing and Imaging ,The Netherlands, June 2005.

[2] Just Kjeldgaard Pedersen, Simon, “ Circular Hough Transform.” Aalborg University, Vision, Graphics, and Interactive Systems. November 2007.

[3] B. Jahne, H. Scharr, and S. Körkel, "Principles of filter design,'In Handbook of Computer Vision and Applications. Academic Press, 1999.

[4] Bresenham, J. E. (1), “Algorithm for computer control of a digital plotter,” IBM Systems Journal, pp25-30 January 1965.

[5] Bies, Robert R, Muldoon, Matthew F., Pollock, Bruce G, Manuck, Steven, Smith, Gwenn, Sale, Mark E, "A Genetic Algorithm-Based, Hybrid Machine Learning Approach to Model Selection,"Journal of Pharmacokinetics and Pharmacodynamics, pp 196-221, 2006.
[6] G. Eason, B. Noble, and I. N. Sneddon, "On certain integrals of Lipschitz-Hankel type involving products of Bessel functions," Phil. Trans. Roy. Soc. London, vol. A247, pp. 529-551, April 1955.

[7] G. Kendall and G. Whitwell, "An evolutionary approach for the tuning of a chess evaluation function using population dynamics," In Proceedings of the 2001 Congress on Evolutionary Computation, pp 995-1002. IEEE Press, World Trade Center, Seoul, Korea, 2001.

[8] Shah, S.M.; Thaker, C. S, Singh, D, “ Multimedia based fitness function optimization through evolutionary game learning,"Emerging Trends in Networks and Computer Communications (ETNCC), 2011 International Conference, page(s): 164- 168,2011.

[9] H.A.Rowley, S.Baluja, T.Kande, "Rotation invariant neural network-based face detection," Computer Science Technical Report, CMU-CS-97-201, CMU, Pittsburgh, 1997.

[10] H.Schneiderman, "T.Kande. Probabilistic modeling of local appearance and spatial relationships for object recognition,"IEEE Conference on Computer Vision and Pattern Recognition, 45-51, Santa Barbara, 1998.

[11] T.K.Leung, M.C.Burl, P.Perona. "Finding faces in cluttered scenes using random labeled graph matching," International Conference on Computer Vision, p: 637-644, Cambridge, MA, 1995. 